\title{
REVIEW OF RECENT DEVELOPMENTS IN CONE-BEAM CT RECONSTRUCTION ALGORITHMS FOR LONG-OBJECT PROBLEM
}

\author{
KAI ZENG AND ZHIQIANG CHEN \\ Tsinghua University, Department of Engineering Physics, Beijing China \\ e-mail: zengkai@tsinghua.org.cn<zengkai@tsinghua.org.cn \\ (Accepted February 13, 2004)
}

\begin{abstract}
Long-object problem and short-object problem both deal with reconstruction problems with truncated conebeam CT projections acquired with a helical path. They have significantly less practical limitations than original exact cone-beam CT reconstruction algorithms which the cone-beam must cover the whole object. The short-object problem can be defined as reconstruction of the whole object having a finite support in the axial direction with helical scan extends a little bit above and below the object's support. However the longobject problem is to reconstruct the central region of interest (ROI) of a long object having an infinite support in the axial direction with helical scan extends a little a bit above and below the ROI. Although the short-object problem is more difficult to solve than the conventional exact reconstruction with non-truncated projections, the long-object problem presents greater challenge to researchers. Recently, with the great development of panel detector technology and computer technology, more and more researchers have been inspired to work on it. Because of great practical value of long-object algorithms, this paper focuses on the review and discussion of recent developments in long-object algorithms. All Long-object algorithms are classified as exact and approximate algorithms. After going briefly over the history of cone-beam algorithms, some novel cone-beam long-object algorithms are introduced, such as: Tam's algorithm, PImethod, PHI-method, etc. Then, the methods described are being compared and discussed.
\end{abstract}

Keywords: cone-beam, CT, image reconstruction, long-object.

\section{INTRODUCTION}

\section{What are long-object problem and short- object problem?}

Short-object problem and long-object problem both aim at reconstruction problems with truncated cone-beam projections (See Fig. 1) acquired with a helical path. The short-object problem is to reconstruct a whole object having a finite support in the axial direction with helical scan that extends a little bit above and below the object's support. Contrary to short-object problem, the long-object problem is to reconstruct the central region of interest (ROI) of a long object having an infinite support in the axial direction with helical scan extends a little a bit above and below the ROI. The short-object problem requires covering the whole object by the scan orbit. But the long-object problem doesn't. Although the short-object problem is more difficult to solve than the conventional exact reconstruction with nontruncated projections, the long-object is rather more difficult than it is. (See Fig. 2)

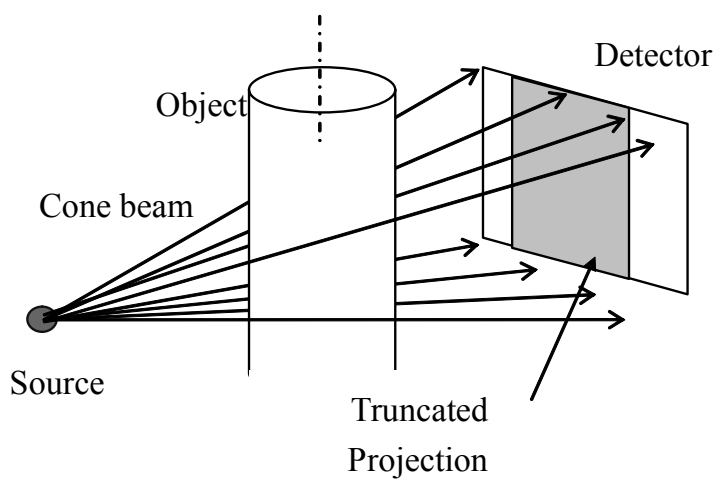

Fig 1. The projection is truncated because the cone beam can't cover the whole object. 


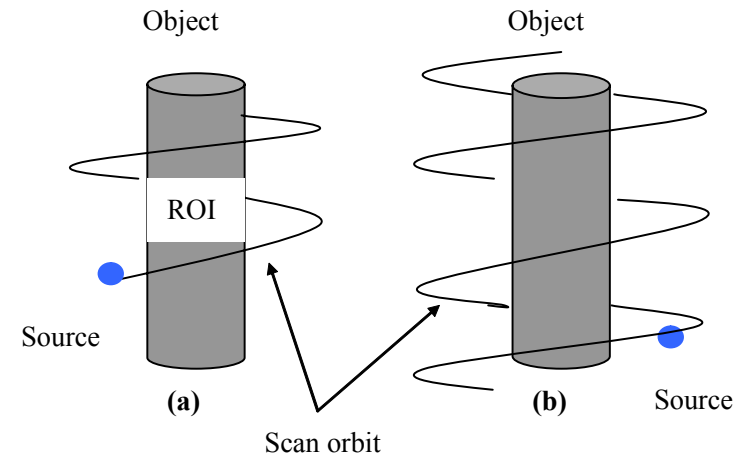

Fig 2. (a) Long-object problem. We can reconstruct the ROI exactly only through scanning part of the object. (b) Short-object problem. In order to reconstruct the object exact, we must scan the whole object.

As long-object problem has fewer limitations, long-object algorithms are more practical than other ones and seem to be very promising in the near future. This paper discusses the recent development in longobject algorithms that are classified as approximate algorithms and exact algorithms. We will introduce some approximate algorithms such as Generalized FDK, PI-method and some exact algorithms such as Tam's algorithm, PHI-method, Kudo's algorithm. Then we will compare and discuss them.

\section{QUICK REVIEW OF CONE-BEAM ALGORITHMS}

As cone-beam CT is able to achieve higher longitudinal resolution and relatively higher volume scan speed, many researchers have been working on it for the last two decades. The first algorithm has been proposed by Feldkamp (Feldkamp et al., 1984), and targets the single circle data acquisition geometry. When imaging large objects, the cone angle becomes larger, and in this case Feldkamp's algorithm degrades severely. To address the problem many researchers developed their own analytical exact reconstruction formulas (Tuy, 1983; Smith, 1985; Zeng et al., 1992; 1994; Axelsson et al., 1994;Wang et al., 1999). The cornerstone of these methods is a relation between the cone beam projection and the 3D Radon transform of the object (Grangeat, 1990). The main limitation of these algorithms derived from Grangeat's is the requirement that their measured projections should not be truncated. Since 1995 two ways (Tam et al., 1997; Kudo et al., 1998) have been proposed to handle truncated projection data. And a few researchers applied this approach to the helical path to obtain two different reconstruction algorithms. However, as all of the algorithms above can't solve long-object problem, they are still classified as short object algorithms. In fact the real, i.e, exact longobject algorithms, have been just developed recently.

\section{RECENT ALGORITHMS FOR LONG OBJECT}

\section{a. Approximate algorithms}

Approximate algorithms generally have the virtue of being computationally less expensive than exact methods. These assets outweigh the approximate character of them when the cone angle is not large, i.e., fewer than 10 degrees. And they are the dominant algorithms, which are used in current commercial helical CT systems.

Rebinning algorithms (MSCT): One kind of approximate algorithms is rebinning approach ( $\mathrm{Hu}$, 1999; Schaller et al., 2000a; 2000b), widely implemented in Multi-Slice CT systems since 1990s. Multi-row detectors are introduced to these systems where cone-beam projections are rebinned into a stack of fan-beam projections corresponding to each slice. For these small cone angles, it has been shown that 2-D filtered back-projection methods combined with axial interpolation provide sufficient image quality.

Generalized FDK algorithm: Another kind of approximate algorithms is based on the FDK algorithm, which was generalized to helical scan by Ge Wang (Wang et al., 1993; 2000). This solution is heuristic derivation of 2-D fan beam reconstruction algorithm. It mainly follows three steps below: each projection are firstly weighted to compensate its deviation from the middle plane, then the row-by-row ramp filtering on the detector plane is applied and, at last, each projection is backprojected into the object space.

PI-methods: Recently a new family of algorithms is developed by Danielsson, Turbell, etc. In the PImethods (Turbell, 2000)' the detector fits exactly the Tam-window, the data capture is complete and almost non-redundant. The discovery of the original PI-method (Danielsson et al., 1997) was based on the simple insight that the rebinned ray geometry guarantees that the backprojection step will deliver the same number of contributions to all object points. The PI-original algorithm consists of the following four steps:

1. Pre-weighting of detector data with cosine of the cone angle

2. Rebinning to obliquely parallel projection data on the virtual detector 
3. Ramp filtering of rebinned data row-by-row

4. Backprojection along the original ray paths without magnification factor.

Tam also developed several algorithms to achieve better image quality or faster speed. (Danielsson et al., 1997;Proksa, 2000)

b. Exact algorithms

However, due to approximate algorithms' intrinsic approximation, they introduce artifacts, which generally rise with increasing the cone angle of cone beam. (See Fig. 1) In contrast to approximate algorithms, exact methods avoid intrinsic approximations and can therefore provide superior image quality for large cone angle.

Tam's algorithm (1998): The first exact long object algorithm for helical scan is has been proposed by Tam (Tam, 1995; 1998; 2000), It requires additionally a top and a bottom circle scans (See Fig. 3). Its idea is to calculate the 3D Radon transform for a given plane, not from a single projection as with the original Grangeat formula but rather from a combination of several projections that provide a kind of triangulation of the plane. It consists of four steps which is a generalization of the six-step CB backprojection technique developed by Kudo (1998). Tam has also developed a combination method by combining PSF filtering with four-step algorithm in order to achieve good image quality and fast speed.

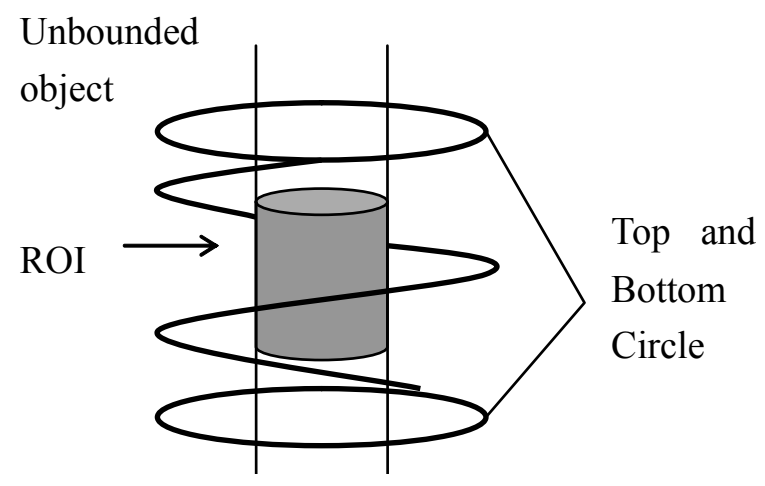

Fig. 3 circles + spiral scan.

Kudo's algorithm (2000): Unfortunately, the above method is not very attractive for practical applications because it requires additional two circles to the helical vertex path. Kudo, etc developed a new long object algorithm (Kudo et al., 1996; 1999; 2000)
(Defrise et al., 2000), based on helical scan only. (See Fig. 4) As this algorithm is derived from an exact formula by introducing very minor approximation, it can be called as quasi-exact long-object algorithm. As a FBP (Filter Backprojection) type algorithm, projection data can be processed parallel. One limitation is that when Ro (the radius of object): Rah (the radius of the helix) $>1.6$, the algorithm will become ineffective. Fortunately in current $\mathrm{CT}$ systems Ro: Rh always much smaller than 1.6, so this algorithm is practical.

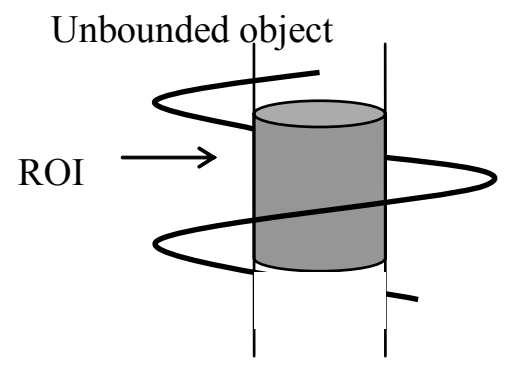

Fig. 4. Pure helcal scan.

PHI-methods (2000): In 2000 Schaller, etc also proposed an exact solution (PHI-method) (Schaller et al., 2000c) to solve long-object problem. The main novelty of the PHI-method is the introduction of a virtual object $f_{\varphi}(x)$ for each value of the azimuthal angle $\varphi$ in the image space, with each virtual object having the property of being equal to the real object $\mathrm{f}(\mathrm{x})$ in some $\operatorname{ROI}(\Omega \mathrm{m})$. And for each $\varphi$, one can calculate exact Radon data corresponding to the twodimensional parallel-beam projection of $\mathrm{f}_{\varphi}(\mathrm{x})$ onto the meridian plane of angle $\varphi$. Then the $\operatorname{ROI}(\Omega \mathrm{m})$ can be exactly reconstructed because $\mathrm{f}(\mathrm{x})$ is identical to $\mathrm{f}_{\varphi}(\mathrm{x})$ in $\Omega \mathrm{m}$. To improve the performance of the PHImethod, attempts to increase the size of the ROI for a given scan range have been considered.

Katsevich algorithm (2002): Recently some further works (Katsevich, 2002) have been made to improve the effectiveness of exact long object algorithm. This newly developed algorithm requires smaller detector, faster reconstruction speed and less restriction to Ro/Rh. As it implements the projection data in PI-line to reconstruct the object, Katsevish's algorithm has better temporal resolution than other algorithms discussed above. 


\section{CONCLUSION}

The main idea of approximation is implement 2-D backprojection through rebinning step, which rebinned projection data into either parallel beam or fan beam. However, exact algorithms aim at calculating the Radon transform of the object. Several approaches have been proposed to handle it such as Tam's a combination of several projections which provide a kind of triangulation of the plane to calculate Radon transform, Schaller's virtual object method and so on. After getting the Radon transform, we can use inverse Radon transform to reconstruct the object. Exact algorithm processed in $3 \mathrm{D}$ space requires more time than approximate algorithm. But its reconstruction quality is better than approximate algorithm especially when the cone angle becomes larger.

Although now all the commercially available helical CT systems implement approximate algorithms, exact long-object algorithms are still promising. Because fast data acquisition is important for increasing patient throughput in screening studies, reducing motion and respiratory artifacts and making good use of the available sustained X-ray power. Fast data acquisition means large helix pitch. And large helix pitch means large cone angle. But approximate algorithms introduce artifacts, which generally become severe with increasing cone angle, due to their intrinsic approximation. Only exact long-object algorithms can handle large cone angle properly. In the near future, exact long-object reconstruction will gain wide implementation when they can process projection data in a tolerable time with the great development of computer technology.

\section{REFERENCES}

Axelsson C, Daniellsson PE (1994). Three-dimensional reconstruction from cone-beam data in $\mathrm{O}(\mathrm{N} 3 \log \mathrm{N})$ time. Phys Med Biol 39:477-91.

Danielsson PE, Edholm P, Eriksson J, Magnusson SM (1997). Towards exact reconstruction for helical conebeam scanning of long object: A new detector arrangement and a new completeness condition, Proc. 1997 Meeting on fully 3d image reconstruction in radiology and nuclear medicine 141-4.

Defrise M, Noo F, Kudo H (2000). A solution to the longobject problem in helical cone-beam tomography. Phys Med Biol 45:623-43.

Feldkamp LA, Davis LC, Kress JW (1984). Practical conebeam algorithm. J Opt Soc Am A 1(1):612-9.

Grangeat P (1990). Mathematical framework of cone beam 3 -D reconstruction via the first derivative of the radon transform.. Mathematical Methods in tomography. Springer Verlag.

Hu H (1999). Multi-slice helical CT: Scan and Rreconstruction. Med Phys 26(1):5-18.

Katsevich A (2002). Improved exact FBP algorithm for spiral CT.

http://www.ai.mit.edu/people/bkph/courses/papers/

Kudo H, Saito T (1996). Extended cone-beam reconstruction using radon transform. IEEE Nuc Sci Symposium, Conference Record 3:1693-7.

Kudo H, Noo F, Defrise M (1998). Cone-beam filteredbackprojection algorithm for truncated helical data. Phys Med Biol 43:2885-909.

Table 1. Comparison between long-object cone-beam CT reconstruction algorithms.

\begin{tabular}{|c|c|c|c|c|c|}
\hline & $\begin{array}{l}\text { Typical } \\
\text { algorithms }\end{array}$ & $\begin{array}{l}\text { Exact or approximate } \\
\text { reconstruction }\end{array}$ & Scan locus & FBP algorithm & Geometrical limitation \\
\hline \multirow[t]{3}{*}{$\begin{array}{l}\text { Approximate } \\
\text { long-object } \\
\text { algorithms }\end{array}$} & $\begin{array}{l}\text { Rebinning } \\
\text { algorithms } \\
(\mathrm{MSCT})\end{array}$ & $\begin{array}{l}\text { Approximate } \\
\text { reconstruction }\end{array}$ & Helix & $\begin{array}{c}\text { Yes } \\
\text { (2D back-projection) }\end{array}$ & None \\
\hline & $\begin{array}{l}\text { Generalized } \\
\text { FDK }\end{array}$ & $\begin{array}{l}\text { Approximate } \\
\text { reconstruction }\end{array}$ & Helix & $\begin{array}{c}\text { Yes } \\
\text { (3D back-projection?) }\end{array}$ & None \\
\hline & PI-methods & $\begin{array}{l}\text { Complete and non- } \\
\text { redundant data capture, } \\
\text { approximate } \\
\text { reconstruction }\end{array}$ & Helix & $\begin{array}{c}\text { Yes } \\
\text { (3D back-projection?) }\end{array}$ & $\begin{array}{c}\text { Detector larger than } \\
\text { Minimize detector (Tam, } \\
\text { 1997) }\end{array}$ \\
\hline \multirow[t]{3}{*}{$\begin{array}{c}\text { Exact long- } \\
\text { object algorithms }\end{array}$} & Tam’s & Exact reconstruction & $\begin{array}{l}\text { Two circles } \\
+ \text { helix }\end{array}$ & $\begin{array}{c}\text { Yes } \\
\text { (3D back-projection?) }\end{array}$ & $\begin{array}{l}\text { Detector larger than } \\
\text { Minimize detector }\end{array}$ \\
\hline & Kudo’s & $\begin{array}{l}\text { Quasi-exact } \\
\text { reconstruction }\end{array}$ & Helix & $\begin{array}{c}\text { Yes } \\
\text { (3D back-projection?) }\end{array}$ & $\begin{array}{l}\text { Detector larger than } \\
\text { Minimize detector; } \\
\mathrm{R}_{\text {object }}: \mathrm{R}_{\text {orbit }}<2: 3\end{array}$ \\
\hline & PHI-method & Exact reconstruction & Helix & No & $\begin{array}{l}\text { Detector larger than } \\
\text { Minimize detector }\end{array}$ \\
\hline
\end{tabular}


Kudo H, Park S, Noo F, Defrise M (1999). Performance of quasi-exact cone-beam filtered back projection algorithm for axially truncated helical data. IEEE Trans Nuc Sci 46(3):608-17.

Kudo H, Noo F, Defrise M (2000). Quasi-exact filtered backprojection algorithm for long-object problem in helical cone-beam tomography. IEEE Trans Med Imag 19(9):902-21.

Proksa R, Kohler TH, Grass M, Timmer J (2000). The nPI-method for helical cone-beam CT. IEEE transactions on medical imaging 19(9):848-63.

Schaller S, Flohr T, Klingenbeck K, Krause J, Fuchs T, A.Kalender W (2000a). Spiral interpolation algorithm for multislice spiral CT-Part I: Theroy. IEEE Trans Med Imag 19(9):822-34.

Schaller S, Flohr T, Klingenbeck K, Krause J, Fuchs T, A.Kalender W (2000b). Spiral interpolation algorithm for multi-slice spiral CT-Part II: Measurment and evalution of slice sensitivity profiles and noise at a clinical multi-slice system. IEEE Trans Med. Imag 19(9):835-47.

Schaller S, Noo F, Sauer F, Tam KC, Flohr T (2000c). Exact Radon Rebinning Algorithm for the long object problem in helical cone-beam CT. IEEE Trans Med Imag 19(5):361-75.

Smith BD (1985). Image reconstruction from cone-beam projection: necessary and sufficient condition and reconstruction methods. IEEE Trans Med Imag MI4:14-28.

Tam KC (1995). Helical and circle scan region of interest computerized tomography. U S Pat 5463 666, OCT 31 .
Tam KC, Samarasekera S, Sauer F (1997). Exact conebeam CT with a spiral scan. Proc. 1997 Meeting on Fully 3D image Reconstruction in Radiology and Nuclear Medicine, 48-51.

Tam KC, Samasekera S, Sauer F (1998). Region-of interest cone beam CT with a spiral scan. SPIE 3336:274-83.

Tam KC, Lauritsch G, Sourbelle K, Ladendorf B (2000). Exact (spiral + circles) scan region-of-interest cone beam reconstruction via backprojection. IEEE Trans Med Imag 19(5):376-83.

Turbell H (2000). Cone beam reconstruction using filtered back projection, Linkopoing strdies in science and technology dissertation no: 672 .

Tuy HK (1983). An inversion for cone-beam reconstruction. SIAM Appl Math 43(2):546-52.

Wang G, Lin TH, Cheng PC, Shinozaki DM (1993). A general cone-beam reconstruction algorithm. IEEE Trans on Med Imag 12(3):486-96.

Wang X, Ning R (1999). A cone-beam reconstruction algorithm for circle-plus-arc data-acquisition geometry. IEEE Trans Med Imag 18(9):815-24.

Wang G, Liu TH (2000). Generalized Feldkamp image reconstruction from equiangular cone-beam projection data. Proceedings of $13^{\text {th }}$ IEEE symposium on 2000 , CBMS 123-8.

Zeng GL, Gullberg GT (1992). A cone-beam tomography algorithm for orthononal circle-and-line orbit. Phys Med Biol 37:563-77.

Zeng GL, Clack R, Gullberg GT (1994). Implantation of Tuy's cone-beam inversion formula. Phys Med Biol 39:493-507. 